\title{
DYSLIPIDEMIA IN PREECLAMPSIA - RISK FACTOR FOR FUTURE MATERNAL CARDIOVASCULAR DISEASES
}

Josephine Latha Pushparaj, Ganesan Subramanyam

1. Assistant Professor, Department of Biochemistry, K.A.P.V. Government Medical College, Tiruchirappalli, Tamil Nadu, Dr. M.G.R. Medical University.

2. Assistant Professor, Department of Biochemistry, K.A.P.V. Government Medical College, Tiruchirappalli, Tamil Nadu, Dr, M.G.R. Medical University.

\section{CORRESPONDING AUTHOR}

Dr. Josephine Latha Pushparaj

New no: 25 / old no: 6b,

Warners road, Cantonment,

Tiruchirappalli- 620 001, Tamilnadu.

E-mail: lathapasel@gmail.com, josephine.latha@yahoo.co.in ,

Ph: 00919486327979.

ABSTRACT: Preeclampsia is still one of the leading causes of maternal and fetal morbidity and mortality. It is associated with insulin resistance \& increase in Free Fatty Acids and plasma Triglycerides well above that seen in normal pregnancy. The pattern of dyslipidemia seen in preeclampsia may predispose these women to future cardiovascular diseases. The aim of this study was to compare the alteration in lipid profile and also the calculated risk ratios of women who developed Preeclampsia with normotensive pregnant women. This cross sectional study was carried out in Mahatma Gandhi Memorial Government Hospital, Trichy, Tamilnadu.100 women with preeclampsia and 100 normotensive pregnant women as controls were included in the study after obtaining their informed consent. The diagnosis of Preeclampsia was done as per the norms of American college of Obstetrics and Gynecologists. Fasting blood samples were collected. Serum glucose, cholesterol, triglycerides, and HDL-C were estimated by standard methods using standard kits. LDL-C was calculated by Friedwald's equation. All the results were analyzed statistically using ANOVA technique. There was significant elevation of total cholesterol, triglycerides, LDL, VLDL and risk ratios TC/HDL and TGL/HDL and decrease in HDL in Preeclamptic group compared to normotensives $(\mathrm{p}<0.0001)$. The dyslipidemic pattern of elevated TGL and reduced HDL which was also reflected in highly elevated TGL/HDL seen in preeclamptic patients is definitely atherogenic and may lead to future maternal cardiovascular disease. Hence simple measurement of lipid profile at 20 weeks of gestation can help in preventing the complications of PIH and future cardio vascular risk of the mother. Further studies in larger population in various ethnic groups are required to predict maternal future cardiovascular risk and its prevention by lifestyle modifications and therapy.

KEY WORDS: Preeclampsia, dyslipidemia, atherogenesis, lipid profile, placental ischemia, cardiovascular disease.

INTRODUCTION: Preeclampsia is still one of the leading causes of maternal and fetal morbidity and mortality. The incidence of preeclampsia ranges from $5-15 \%$ (1). In India the incidence of preeclampsia is reported to be $8-10 \%$ of the pregnancies (2). The incidence in primigravidae is about $10 \%$ and in multigravidae about $5 \%$ (3). It causes IUGR leading to low birth weights. Low birth weight child is prone to suffer from diabetes, hypertension, and coronary vascular disorders in their later life(1). Complications of Preeclampsia are HELLP syndrome (4), Renal 
failure / Impairment, Pulmonary edema, Disseminated intravascular coagulation, Eclampsia and Placental abruption. According to the norms of American college of obstetrics and Gynecologist, the diagnostic criteria for Pregnancy Induced Hypertension (PIH) (5) is 1) Systolic Blood pressure $\geq 140 \mathrm{~mm} / \mathrm{Hg}$ 2) Diastolic Blood pressure $\geq 90 \mathrm{~mm} / \mathrm{Hg}$ Or 3 ) increase of $\geq 30 \mathrm{~mm} / \mathrm{Hg}$ in Systolic pressure Or 4) increase of $\geq 15 \mathrm{~mm} / \mathrm{Hg}$ in Diastolic pressure, in a previously normotensive woman. Preeclampsia usually starts after 20 weeks of pregnancy and is defined as a gestational blood pressure elevation $(>140 / 90 \mathrm{mmHg})$, combined with proteinuria $(>300$ $\mathrm{mg} / 24 \mathrm{~h}$, or a positive dipstick $30 \mathrm{mg} / \mathrm{dl}$ ).

The blood pressure is normally determined by Cardiac output and Peripheral vascular resistance (1). In normal pregnancy, though cardiac output is increased (7) blood pressure is maintained because of the decrease in peripheral vascular resistance. But in PIH there is increased resistance due to the increased response to vasopressors(6), altered lipid synthesis leading to decrease in the ratio of $\mathrm{PGI}_{2} / \mathrm{TXA}_{2}$ and antioxidants/lipid peroxides(6) and changes in the local factors like NO, endothelins.

There is an accumulated evidence stating that abnormal placentation is one of the initial events leading to this disease. Impaired interstitial trophoblasts invasion and failure of vascular invasion leading to inadequate perfusion of feto-placental unit along with Impaired decidual remodeling,(7) Impaired function of uterine natural killer cells and maternal endothelial failure to express adhesion molecules and reduced placental L-arginine concentration due to excessive arginase II expression decrease the production of nitric oxide. This promotes abnormal placental perfusion and microvascular oxidative damage. Defective placental implantation causes placental ischemia leading to endothelial dysfunction (7). This leads to Reduced perfusion of affected organs (Predominantly kidneys, liver \& brain) that leads to the Clinical manifestation of Preeclampsia.

Normal pregnancy is hyperlipidemic (i.e.) 3 fold increase in TGL and fatty acids, 50\% increase in LDL and HDL. One of the reasons for this increase may be due to the reduction in intestinal motility. Reduction of enterohepatic circulation with increased excretion of cholesterol in the bile also leads to alteration in lipid profile ${ }^{(8)}$. Normal pregnancy is also a state of hyperestrogenemia. Estrogen results in increase in HDL level and TGL level, decrease in LDL level.

Pre-eclampsia is associated with insulin resistance \& increase in FFA (antedating clinical expression of the disorder) and plasma TGL well above that seen in normal pregnancy(Lorenzen et al 1995) ${ }^{8}$.Hypoestogenemia, predominance of smaller and denser serum LDL and significant concentration of soluble vascular cell adhesion molecule-1 are important contributors for endothelial dysfunction in Preeclampsia (6).

In lipid mediated endothelial dysfunction an essential step is oxidation of low-density lipoprotein (9-11). The atherogenesis itself may be initiated by hypertriglyceridemia. Hypertriglyceridemia leads to elevated level of small dense LDL particles which is atherogenic. Since Triglyceride related vasculopathy may influence a future pregnancy as well as a woman's long term risk of cardiovascular disease, it is worthwhile to explore the metabolism and transport of various sub fractions of lipoproteins in pregnant women from 20 weeks of gestation who have developed increase in blood pressure. Early identification of women at risk for PIH may help in preventing the complications of the disease. This study aims at comparing the alteration in lipid profile and the cardiovascular risk ratios of women who developed Preeclampsia with normotensive pregnant women. 
MATERIALS AND METHODS: This cross sectional study was carried out in Mahatma Gandhi Memorial Government Hospital, Trichy, Tamilnadu.100 women with preeclampsia and 100 normotensive pregnant women as controls were included in the study after obtaining their informed consent. The diagnosis of Preeclampsia was done as per the norms of American college of Obstetrics and Gynecologists. All the participants were inquired by a questionnaire containing their personal history, family history of PIH, Twins, Hypertension and Diabetes, Drug history and their symptoms.

The study included individuals of any gravida of age between $20 \mathrm{yrs}$ and $45 \mathrm{yrs}$, after 20 wks of pregnancy with Blood Pressure $\geq 140 / 90$ and proteinuria (positive dipstick) for preeclampsia and individuals with Blood Pressure $\leq 120 / 80$ for control. Subjects with history of Diabetes, Hypertension, Hyperlipidemia before 20 wks and Edema, Proteinuria, oliguria, Hepatic disease and Involvement of other Organs were excluded. Clinical examination of participants were carried out to rule out Diabetes, Hypertension, Hyperlipidemia before 20 wks, edema, proteinuria, oliguria, Hepatic disease, involvement of other Organs..

Fasting blood samples were collected; the serum was separated and analyzed for the following parameters.

The automated analytical system applying routine methods was used for the following measurements: glucose (GOD-POD method), TC (cholesterol esterase method), and HDL-C after precipitation using phosphotungstate method (cholesterol esterase method), and triglyceride (lipase method). LDL-C was calculated by using the Friedwald equation [LDL-C = TC - (HDL-C + triglyceride/5)], where the triglyceride level was less than $400 \mathrm{mg} / \mathrm{dL}$. The risk ratios were calculated from the estimated values. All the results were tabulated. $24 \mathrm{hrs}$ urine collected and protein estimated by analysis using Roche URS-345 dipstick urinalysis stripe to diagnose preeclampsia.

The various results obtained were statistically analyzed using ANOVA technique. Correlations between the variables were estimated by Pearson's correlation coefficients. Significance was assumed if the $P$ value was less than 0.05 .

RESULTS: The values of the lipid fractions were tabulated as mean \pm SD (Table 1). Total cholesterol, triglycerides, LDL-C, VLDL-C were significantly elevated and HDL-C was significantly decreased in Preeclampsia group compared to control $(\mathrm{p}<0.0001)$.

Table 1

\begin{tabular}{|l|l|l|l|}
\hline Test & Preeclampsia & Control & p value \\
\hline TC & $209.54 \pm 32.46$ & $175.16 \pm 30.16$ & $<0.0001$ \\
\hline TGL & $337.24 \pm 77.29$ & $197.48 \pm 37.95$ & $<0.0001$ \\
\hline HDL & $37.62 \pm 7.71$ & $47.50 \pm 7.90$ & $<0.0001$ \\
\hline LDL & $104.47 \pm 34.62$ & $88.164 \pm 30.22$ & 0.0006465 \\
\hline VLDL & $67.45 \pm 15.46$ & $39.496 \pm 7.60$ & $<0.0001$ \\
\hline
\end{tabular}

(TC-Total cholesterol, TGL-Triglycerides, HDL-High density cholesterol, LDL-Low density cholesterol, VLDL-Very Low density cholesterol)

The risk ratios TC/HDL, TGL/HDL, and LDL/HDL were significantly elevated and HDL/VLDL significantly decreased in Preeclampsia group compared to control $(\mathrm{p}<0.0001)$ (Table 2). The results of various lipid sub-fractions and the atherosclerotic risk ratios were 
compared between the preeclampsia and control groups and shown in FIGURE 1 and FIGURE 2 respectively.

Table 2

\begin{tabular}{|l|l|l|l|}
\hline Test & Preeclampsia & Control & p value \\
\hline TC/HDL & $5.843 \pm 1.74$ & $3.81 \pm 0.99$ & $<0.0001$ \\
\hline TGL/HDL & $9.41 \pm 3.27$ & $4.30 \pm 1.21$ & $<0.0001$ \\
\hline HDL/VLDL & $0.58 \pm 0.17$ & $1.26 \pm 0.37$ & $<0.0001$ \\
\hline LDL/HDL & $2.96 \pm 1.36$ & $1.95 \pm 0.85$ & $<0.0001$ \\
\hline
\end{tabular}

Figure-1

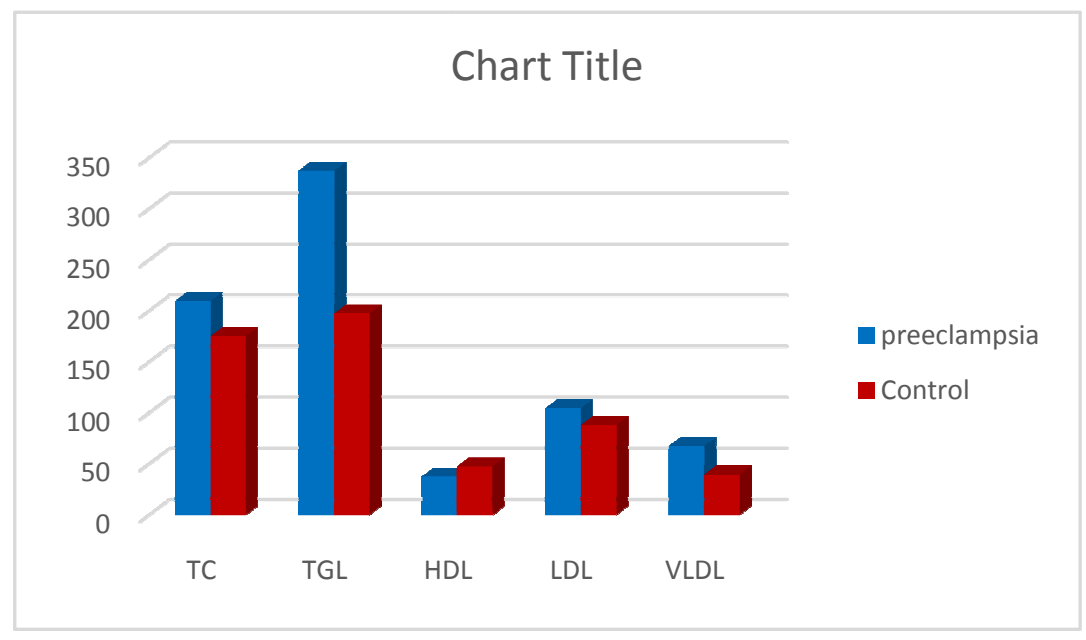

\section{Comparison Of Lipid Profile In Preeclampsia With That Of Control}

Figure-2

LDL/HDL

HDL/VLDL

TGL/HDL

TC/HDL
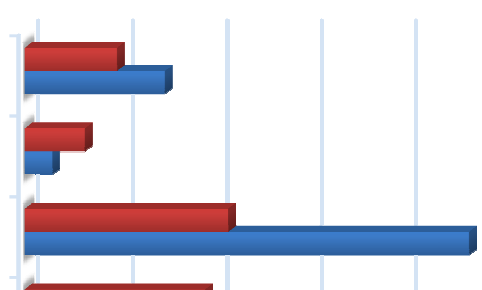

- Control

- preeclampsia

\section{Comparing Calculated Risk Ratios Between Preeclamptic and Control Groups}


The risk ratio TGL/HDL had a significant positive correlation with triglycerides ( $\mathrm{r}=$ $0.762)$ and a negative correlation with HDL-C ( $\mathrm{r}=-0.674$ in patients). The correlation was highly significant $(\mathrm{P}<0.001)$. From factor analysis by rotated component matrix, the 3 factors TGL/HDL, TGL or VLDL \& TC accounted for 89.644 percent of the variance and hence contribute more to the preeclampsia. The significant elevation of Triglycerides and the ratio TGL/HDL in preeclamptic women compared to the normotensive pregnant women can be seen in the following charts.

Figure-3: Triglyceride values in Preeclampsia and Control Group

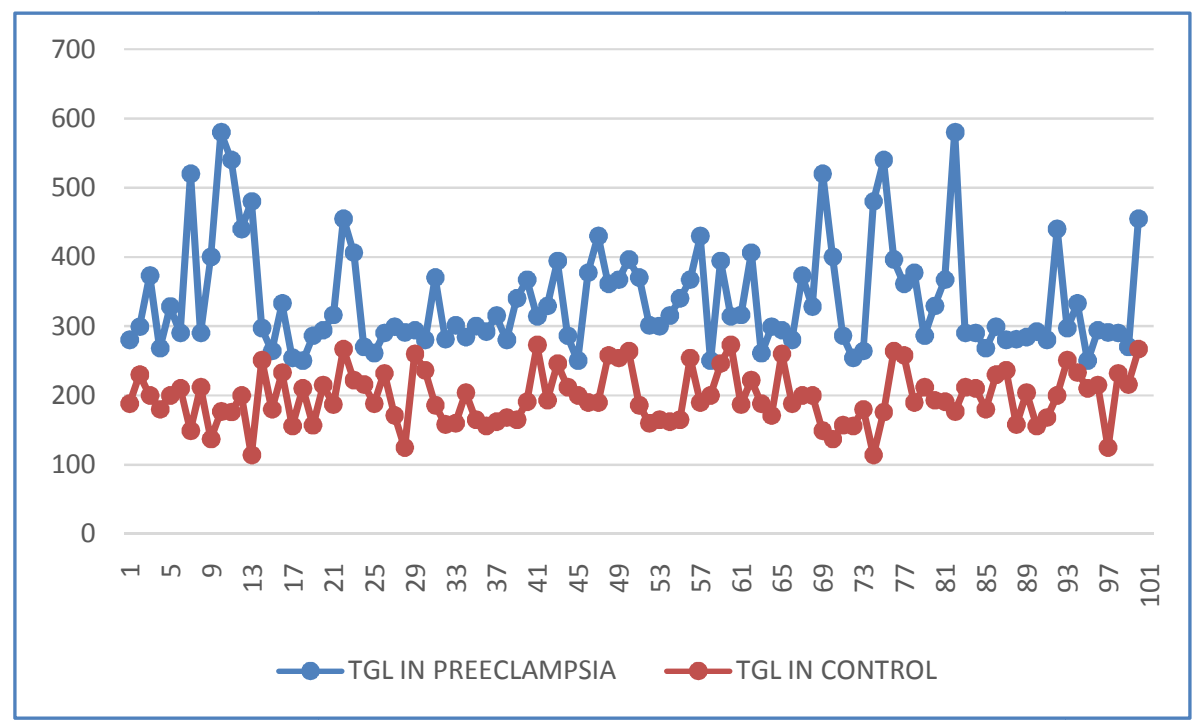

Figure-4: Atherogenic Index(TGL/HDL) In Preeclampsia And Control Group

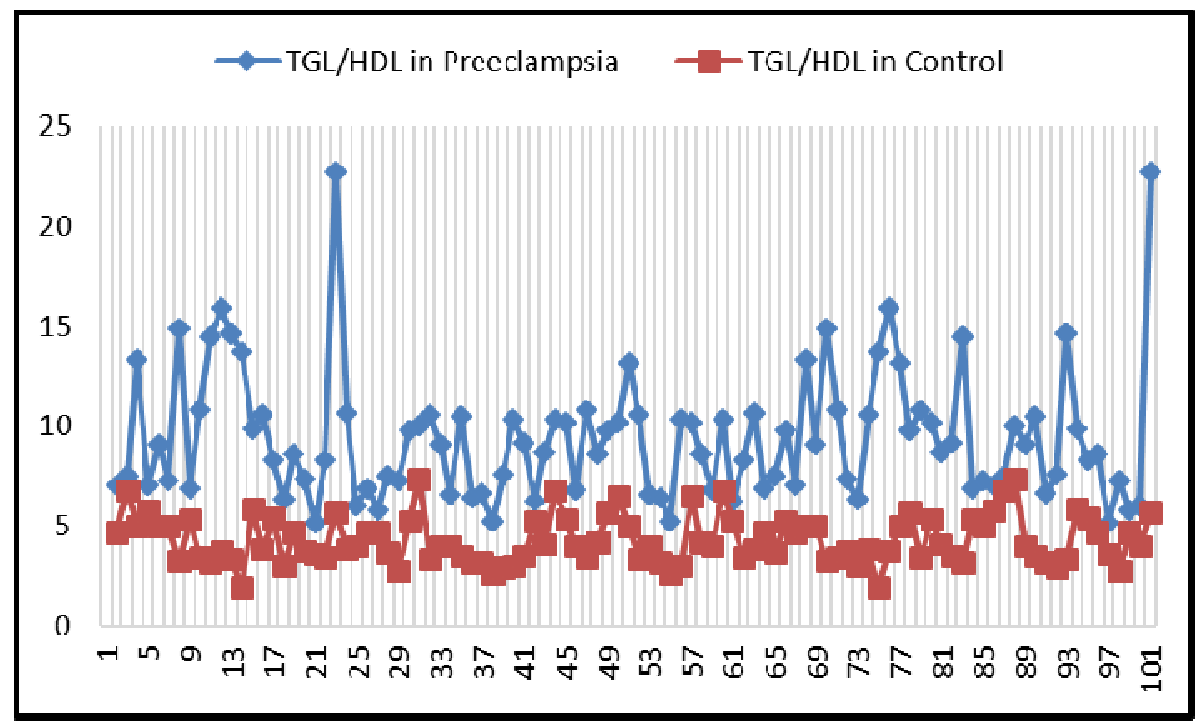


DISCUSSION: There was dramatic alteration in lipid profile which has been elevated since 20 weeks of gestation. In our study all the lipid sub fractions were significantly elevated, more in the case of triglycerides (figure 1) and HDL was significantly decreased. The significant rise in serum triglyceride concentration in pre-eclampsia in our study was established in the studies of many workers $(12,13)$. The exaggerated TGL rise leads to elevated atherogenic small dense low density lipoprotein concentrations (7,14-16). Hepatic lipase activity has been shown to be elevated in PE and could contribute to increased small dense low density lipoprotein concentration (7), via increased TGL exchange into LDL, followed by hepatic lipase induced lipolysis of the particle (17). HDL cholesterol levels are reduced, probably as a consequence of the increased TGL levels and elevated hepatic lipase activity ${ }^{(18) .}$

One of the reasons for this dyslipidemia is due to hormonal imbalance in preeclampsia. Preeclampsia is a state of hypoestrogenemia (6). Decreased utero-placental blood flow which is the main pathophysiological event in preeclampsia leads to impairment in the formation of Dehydroepiandrosterone sulphate (DHEA) by fetal adrenal glands by interfering with the uptake of lipids by the fetus. DHEA is the important source of estrogen in pregnancy, (i.e.) $90 \%$ of estrogen in maternal circulation is from fetal DHEA which on desulphuration and aromatization by the enzymes sulfatase and aromatase in the placenta forms estriol (19). Because of the impairment in the formation of DHEA estrogen levels decrease (6). Moreover decrease in utero-placental blood flow leads to relative stasis of maternal blood in the intervillous space resulting in redistribution of steroids formed in syncytium back to the fetus instead of entering into maternal circulation which also results in a state of hypoestrogenemia(20).

The state of hypoestrogenemia leads to decreased expression of VLDL / apo E receptors in the placenta that are essential for the lipid transport to the fetus. This results in reduced transport of VLDL to fetal compartment, which may be the reason for maternal hypertriglyceridemia. Further LDL taken up by the fetus for the synthesis of DHEA is decreased due to reduced fetoplacental perfusion leading to increased LDL. The decreased catabolism of TG rich lipoproteins by reduced placental uptake and the simultaneous decrease of lipoprotein lipolysis results in the accumulation of TG rich remnant lipoproteins in the maternal circulation $(21,22)$.

The changes in lipoprotein sub fraction seen in our study were compatible with changes seen in coronary artery disease (Kaaja et al 1995, sattar et al 1997). The dramatic increase in TGL(as shown in figure 3) causes increase in VLDL, small changes in LDL and decrease in HDL which finally leads to Vascular damage and dysfunction directly \& increased oxidative stress potentiating insulin resistance - Vascular dysfunction indirectly (Hayman et al 1999\& saltar \& Green 1999) (23).

In the late phase of normal pregnancy, estrogen enhances the production of VLDLs and decreases maternal lipolytic activity (24). Together with the increased expression of the VLDL/apoE receptors in the placenta (25), this may result in a coordinated rerouting of TG-rich lipoproteins from the mother toward the fetoplacental unit to meet the nutritional demands of the growing fetus(26). But in pre-eclampsia, the reduced maternal lipolysis and the hampered uptake of TG-rich lipoproteins by the fetoplacental unit lead to the accumulation of TG-rich remnant lipoproteins in the maternal circulation. Renal protein excretion and hypertension may both reflect endothelial dysfunction. Arbogast et al. (27) hypothesized that TG-rich lipoproteins from preeclamptic women damage the endothelium.

Some studies show that mutation in LPL gene in pre-eclampsia may lead to lowered activity of LPL resulting in decreased hydrolysis of Triglycerides and predispose to dyslipidemia 
and cardiovascular disease (28). There is also evidence that there is active role of placental LPL and Apo E in the metabolism of maternal lipoproteins and so fetal genes may modulate the risk for problems related to maternal dyslipidemia. (29)

The recently published results of the ongoing Copenhagen Male Study, which studied the effect, the TGL/HDL ratio has on the long-term development of heart disease showed that TGL/HDL ratio $>6$ had a much higher risk of developing heart disease(29)(30). This was observed in our study also, (the TGL/HDL ratio in preeclamptic population was 9.41 (high risk) against 4.30 in normal pregnancy). The significant increase of TGL/HDL ratio in preeclamptic population is shown in figure 4. The dyslipidemia of elevated triglycerides and lowered HDL in our study was similar to that of previous studies (31-33).

CONCLUSION: The pattern of dyslipidemia seen in Preeclampsia is similar to the pattern in coronary heart disease. Atherogenic dyslipidemia (elevated triglycerides and low HDL-C) appears to be an important independent risk factor for CVD, confirmed by principal component analysis of lipoprotein subfractions in a large prospective cohort study (34). Elevation in the ratio of TGL to HDL-C was shown to be the single most powerful predictor of extensive coronary heart disease among all the lipid variables (35). From all these facts it is clear that dyslipidemia in pre-eclampsia is definitely predisposing to future maternal cardiovascular disease. Hence simple measurement of lipid profile at 20 weeks of gestation can go a long way in prevention of the complications of PIH like, eclampsia, intrauterine growth retardation, HELLP syndrome future cardio vascular risk of the mother, future development of Hypertension and stroke. Further prospective cohort studies in larger population in various ethnic groups are required to predict maternal future cardiovascular risk and its prevention by lifestyle modifications and therapy in preeclampsia. Women with hypertensive disorders in pregnancy should therefore be monitored more closely for the future development of hypertension and the presence of other cardiac risk factors. Furthermore, the obstetric history may be an important additive tool in the cardiovascular risk evaluation in women with hypertension and/or chest pain syndromes.

\section{REFERENCES:}

1. Carno TA, Nitrini SM. Drug Prescription for Pregnant Women: A pharmacoepidemiological study. Cad Saud Publica 2004; 20: 1004-13.

2. Krishna Menon MK, Palaniappan B. Hypertensive disorders of pregnancy. In: Mudaliar Menon editor. Clinical Obstetrics 9th ed. Madras: Orient Longman; 1994. 133-54.

3. Dutta DC. In: Text Book of Obstetrics. Hiralal K. editor. 6th ed. Calcutta: New central book agency ; 2006:46, 52 .

4. D.J. Williams M. de swiet, Review Article: The Patho physiology of Pre-eclampsia. Intensive Care Med :1997: 23:620-29.

5. F.Gary Cummingham, Norman FG,Kenneth et al. Hypertensive disorders in pregnancy, Williams Obstetrics, 22nd Edition, Mc. Graw Hill 2005:761-64.

6. Jayanta De, Anandhakumar Mukhopadhyay and Pradip Kumar Saha Study of Serum Lipid Profile in Pregnancy Induced Hypertension. Indian Journal of Clinical Biochemistry 2006/21 (2) 165-68.

7. Marina Noris, Norberto Perico and Giuseppe Remuzzi Mechanisms of Disease: Preeclampsia Nature Clinical practice Nephrology 2005:1(2) 98-114.

8. Turnbull's Obstetrics. Geoffrey Chamberlain, Philip J.Steer 3rd Edition. Churchill Livingstone 2001. 
9. Ray J, Diamond P, Singh G, Bell C. Brief overview of maternal triglycerides as a risk factor for pre-eclampsia. BJOG 2006; 113:379-86.

10. Gratacos E, Casals E, Gomez O, Liurba E, Mercader I, Cararach V, et al Increased susceptibility to low density lipoprotein oxidation in women with a history of Preeclampsia.BJOG 2003:110:400-04.

11. Staff AC, Ranleim T, Khoury J, Henriksen T Increased contents of phospholipids, cholesterol and lipid peroxides in Decidua Basalis in women with Preeclampsia Am J Obstet Gynecol 1999:180:587-92

12. Enquobahrie, DA, Williams MA, Butler CL, Frederick IO, Miller RS and Luthy DA. Maternal plasma lipid concentrations in early pregnancy and risk of preeclampsia. Am J Hypertens.2004 July;17(7):574-81

13. Cekmen MB, Erbagci AB, Balat A, Duman C, Maral H, Ergen K, et al. Plasma lipid and lipoprotein concentrations in pregnancy induced hypertension. Clin. Biochem. 2003; 36(7):575-78.

14. Sattar N, Bendomir A, Berry C, et al. Lipoprotein subfraction concentrations in preeclampsia: pathogenic parallels to atherosclerosis. Obstet Gynecol 1997;89: 403-08.

15. Ogura K, Miyatake T, Fukui O, et al. Low-density lipoprotein particle diameter in normal pregnancy and preeclampsia. J Atheroscler Thromb 2002;9: 42-7.

16. Lorentzen B, Henriksen T. Plasma lipids and vascular dysfunction in preeclampsia. Semin Reprod Endocrinol 1998;16:33-9.

17. Belo L, Caslake M, Gaffney D, et al. Changes in LDL size and HDL concentration in normal and preeclamptic pregnancies. Atherosclerosis 2002; 162:425-32.

18. Tan CE, Foster L, Caslake MJ, et al. Relations between plasma lipids and post heparin plasma lipases and VLDL and LDL subfraction patterns in normolipemic men and women. Arterioscler Thromb Vasc Biol 1995;15: 1839-48.

19. Williams Obstetrics. F.Gary Cummingham et al 23rd edition. Mc. Graw Hill 2010:65-70.

20. Frank Hytten, Geoffrey Chamberlain's Clinical physiology in Obstetrics. $2^{\text {nd }}$ edition, Boston: Blackwell Scientific; 1991.

21. Rubina A, Tabassum M. Pre-eclampsia and lipid profile. Pakistan Journal of Medical Sciences. 2007 Oct-Dec (Part-I) ; 23(5): 751-54.

22. Karl W, Birgit W, Michael M. H et al.Triglyceride Rich Lipoproteins Are Associated with Hypertension in Preeclampsia. The Journal of Clinical Endocrinology \& Metabolism 2003;88(3):1162-66.

23. Kaaja R, Tikkanen MJ, Viinikka L et al. Serum lipoprotein, insulin, and urinary prostanoid metabolites in normal and hypertensive pregnant women. Obstet Gynecol 1995;85:353-56.

24. Kinnunen PK, Unnerus HA, Ranta T, Ehnholm C, Nikkila EA, Seppala M 1980. Activities of postheparin plasma lipoprotein lipase and hepatic lipase during pregnancy and lactation. Eur J Clin Invest 10:469 -74.

25. Wittmaack FM, Gafvels ME, Bronner M, Matsuo H, McCrae KR, Tomaszewski JE, Robinson SL, Strickland DK, Strauss III JF 195. Localization and regulation of the human very low density lipoprotein/apolipoprotein-E receptor: trophoblast expression predicts a role for the receptor in placental lipid transport. Endocrinology 136: 340-48.

26. Winkler K, Wetzka B, Hoffmann MM, Friedrich I, Kinner M, Baumstark MW, Wieland H, Marz W 2000 Low Density Lipoprotein(L DL) subfractions during pregnancy: 
accumulation of buoyant LDL with advancing gestation. J Clin Endocrinal Metab 85: 4543-50.

27. Arbogast BW, Leeper SC, Merrick RD, Olive KE, Taylor RN 1994 Which plasma factors bring about disturbance of endothelial function in pre-eclampsia. Lancet 343:340-41.

28. Williams RR, Hunt SC, Hopkins PN, Hasstedt SJ, Wu LL, Lalouel JM. Tabulations and Expectations regarding the genetics of human hypertension. Kidney Int. 1994; $45: 57$ 64.

29. Descamps,O. S., M. Bruniaux, P-F. Guilmot, R. Tonglet, and F. R.Heller.Lipoprotein metabolism of pregnant women is associated with both their genetic polymorphisms and those of their newborn children. J. Lipid Res.2005.46:2405-14.

30. Џørgen Jeppesen, Hans Ole Hein, Poul Suadicani, Finn Gyntelberg Relation of High TGLow HDL Cholesterol and LDL Cholesterol to the Incidence of Ischemic Heart Disease An 8-Year Follow-up in the Copenhagen Male Study. Arteriosclerosis, Thrombosis and Vascular Biology: 1997; 17: 1114-20

31. Vidyabati RK, Hijam Davina, Singh NK, Singh W Gyaneshwar Serum $\beta$ hCG and lipid profile in early second trimester as predictors of pregnancy induced hypertension J Obstet Gynecol India 2010 February : 60(1) 44-50

32. Adiga Usha and Adiga Sachidananda Dyslipidemia In Pregnancy Induced Hypertension Journal of Global Pharma Technology ; 2010:2(5): 69-72

33. Shalini Maksane, Rashmi Ranka, Nitin Maksane and Anjali Sharma Study of Serum Lipid Profile and Magnesium in Normal Pregnancy and in Pre-Eclampsia: A Case control Study Asian Journal of Biochemistry 2011:6 (3): 228-39.

34. Kiran Musunuru Atherogenic Dyslipidemia: Cardiovascular Risk and Dietary Intervention Lipids. 2010 October; 45(10): 907-14.

35. Protasio Lemos da Luz, Desiderio Favarato, Jose Rocha Faria-Neto Junior, Pedro Lemos, and Antonio Carlos Palandri Chagas, High ratio of triglycerides to HDL-cholesterol predicts extensive coronary disease Clinics. 2008 August; 63(4): 427-32. 